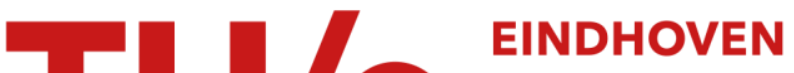

\section{Supervisory control of discrete-event systems under attacks: an overview and outlook}

\section{Citation for published version (APA):}

Rashidinejad, A., Lin, L., Wetzels, B. H. J., Zhu, Y., Reniers, M., \& Su, R. (2019). Supervisory control of discreteevent systems under attacks: an overview and outlook. In 2019 18th European Control Conference (ECC) (pp. 1732-1739). [8795849] Institute of Electrical and Electronics Engineers. https://doi.org/10.23919/ECC.2019.8795849

DOI:

10.23919/ECC.2019.8795849

Document status and date:

Published: 25/06/2019

\section{Document Version:}

Publisher's PDF, also known as Version of Record (includes final page, issue and volume numbers)

\section{Please check the document version of this publication:}

- A submitted manuscript is the version of the article upon submission and before peer-review. There can be important differences between the submitted version and the official published version of record. People interested in the research are advised to contact the author for the final version of the publication, or visit the $\mathrm{DOI}$ to the publisher's website.

- The final author version and the galley proof are versions of the publication after peer review.

- The final published version features the final layout of the paper including the volume, issue and page numbers.

Link to publication

\section{General rights}

Copyright and moral rights for the publications made accessible in the public portal are retained by the authors and/or other copyright owners and it is a condition of accessing publications that users recognise and abide by the legal requirements associated with these rights.

- Users may download and print one copy of any publication from the public portal for the purpose of private study or research.

- You may not further distribute the material or use it for any profit-making activity or commercial gain

- You may freely distribute the URL identifying the publication in the public portal.

If the publication is distributed under the terms of Article 25fa of the Dutch Copyright Act, indicated by the "Taverne" license above, please follow below link for the End User Agreement:

www.tue.nl/taverne

Take down policy

If you believe that this document breaches copyright please contact us at:

openaccess@tue.nl

providing details and we will investigate your claim. 


\title{
Supervisory Control of Discrete-Event Systems under Attacks: An Overview and Outlook*
}

\author{
Aida Rashidinejad ${ }^{1}$, Liyong Lin $^{2}$, Bart Wetzels ${ }^{1}$, Yuting Zhu ${ }^{2}$, Michel Reniers ${ }^{1}$, Rong Su$^{2}$
}

\begin{abstract}
Due to insecure network-based communications, cyber-physical systems face the risks of cyber attacks which might result in catastrophic damage. This paper provides an overview of existing approaches preventing damage caused by cyber attacks in the supervisory control of cyber-physical systems. The objective is to identify missing pieces, possible links and determine new directions for further research to improve the existing literature. For this purpose, first, we classify the current research works under a framework consisting of three dimensions: 1) the communication channel where an attack can happen, 2) the attack impact on the transmitted data, and 3) the mechanism to prevent damage. This classification will then help us to compare the existing techniques and investigate how they can be improved to get closer to the ideal scenario where most kinds of attacks can be handled so that the closed-loop system remains safe.
\end{abstract}

\section{INTRODUCTION}

Cyber-physical systems (CPS) are the integration of computation, networking and physical processes. CPS can be found in many areas varying from manufacturing and chemical processes to aerospace and healthcare systems [1]. Insecure network-based communications between the cyber part and the physical part in CPS raises the need to deal with cyber security, especially for applications where an attack might put the system and human safety at risk, as discussed in [2]-[6] and in [7]-[9] for smart grids and water networks, respectively. For this reason, security of CPS has gained a lot of attention in recent years [10]-[13].

In this paper, we focus on works in the literature investigating cyber-attacks in the supervisory control of a CPS modeled as a discrete-event system (DES). A DES is a dynamical system defined over a discrete set of states with state transitions that are caused by the occurrences of events [14]. Supervisory control theory has been developed for DES to ensure safety and progress properties of the closed-loop control systems [15]. Figure 1 depicts a typical supervisory control system ${ }^{1}$ under adversarial attack. In an active attack, the attacker's goal is to inflict damage on the system. This

* This research has received funding from the European Union's Horizon 2020 Framework Programme for Research and Innovation under grant agreement no 674875, and this work is also financially supported by Singapore Ministry of Education Academic Research Grant RG91/18-(S)SU RONG (VP), which is gratefully acknowledged.

${ }^{1}$ Aida Rashidinejad, Bart Wetzels and Michel Reniers are with Department of Mechanical Engineering, Eindhoven University of Technology, The Netherlands A.Rashidine jadetue.nl

${ }^{2}$ Liyong Lin, Yuting Zhu and Rong Su are with School of Electrical and Electronic Engineering, Nanyang Technological University, Singapore

${ }^{1}$ There are various architectures that are largely incompatible; consequently, it is almost impossible to show all these architectures within a single diagram. Some generalizations and alternatives will also be discussed and explained throughout the paper. is in contrast to a passive attack, where the attacker's goal is to learn secrets about the system [16].

The sensor data gathered from the plant are observed by the supervisor through the observation channel, and control commands are transmitted from the supervisor to the actuators in the plant through the control channel. Notations $\Sigma, \Sigma_{o} \subseteq \Sigma$, and $\Sigma_{c} \subseteq \Sigma$ stand for the set of events executed in the plant, events that are observable by the supervisor and events that are controllable by the supervisor, respectively. The control commands issued by the supervisor are control patterns in $\Gamma$, where $\Gamma:=\left\{\gamma \subseteq \Sigma \mid \Sigma_{u c} \subseteq \gamma\right\}$ and $\Sigma_{u c}=\Sigma \backslash \Sigma_{c}$ denotes the set of uncontrollable events. Both the observation channel and the control channel may be insecure. An attacker can corrupt a subset of events (vulnerable events) transmitted from sensors to the supervisor (observable events) and from the supervisor to the actuators in the plant (controllable events). Observable vulnerable events and controllable vulnerable events ${ }^{2}$ are denoted by $\Sigma_{o v} \subseteq \Sigma_{o}$ and $\Sigma_{c v} \subseteq \Sigma_{c}$, respectively. After being attacked, an observable vulnerable event $\sigma \in \Sigma_{o v}$ or an empty string $\epsilon$ can be replaced by a word $w \in \Sigma_{o v}^{*}$, and a non-vulnerable event stays the same. A control command $\gamma \in \Gamma$ can be replaced by a new control command $\gamma^{\prime} \in \Gamma$ such that $\gamma^{\prime} \backslash \Sigma_{c v}=\gamma \backslash \Sigma_{c v}$. The attacker in Figure 1 has no extra sensors for observing the execution of the plant, as we assume the attacker only listens to the insecure communication channels. In particular, we assume the attacker has full observation on the data transmitted on the insecure communication channels. In addition, we assume the supervisor sends a new control command message to the plant whenever it receives a piece of information, i.e., the occurrence of observable events, from the observation channel. There are many other possibilities. For example, the attacker can place additional sensors for observing the execution of the plant or it has only partial access to the communication channels, or a combination thereof. Also, the supervisor does not need to send a control command each time when it observes an occurrence of observable events. Different assumptions will lead to variations of the architecture given in Figure 1.

In this paper, we shall analyze attack-prevention techniques in supervisory control of CPS represented by DES, that are available in the literature, and pinpoint potential directions for future research. For this purpose, first, we provide a framework to classify the current approaches. This classification enables us to compare the available techniques

${ }^{2}$ For generality, in this work vulnerability is defined on the level of events to accomodate the possiblity of different sub-channels for different events. 


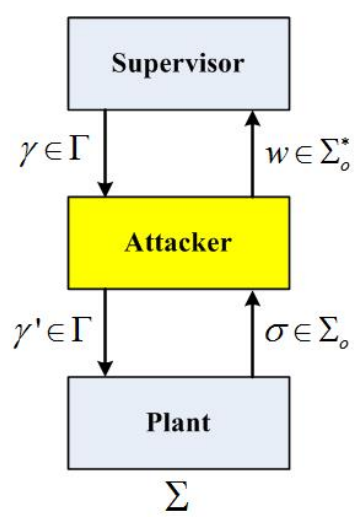

Fig. 1. Supervisory control system under attacks

and find the areas which need further improvements. Moreover, we discuss some relevant works dealing with similar issues in a supervisory control system, such as faults and various communication problems. Finally, we shall outline new research lines and conclude the paper.

\section{FRAMEWORK}

The attacks studied in this framework are mainly active attacks, where the attacker's goal is to inflict damage rather than learning secrets about the system. Based on the works presented in the literature that discuss the security of a supervisory control system under (active) attacks, we classify them based on the following dimensions:

- the attack location,

- the attack impact on transmitted data,

- the security mechanism.

The first two are related to the characteristics of the attacks, while the last one is related to the different mechanisms by which a supervisor can deal with or prevent the attacks. In the next subsections, these three dimensions are discussed in more detail. As we shall see soon, the classification is far from complete and there are many dimensions that we have to omit, due to the limited number of research works in this area. Many aspects that we do not classify are discussed in Section VI, including passive attacks.

\section{A. Attack Location}

As Figure 1 shows, for a closed-loop supervisory control system, an attack may occur in the observation channel (known as a sensor attack), in the control channel (known as an actuator attack) or in both observation and control channels (as the most realistic case) [17], [18]. Due to the different direct impacts that sensor and actuator attacks can make on the system, most papers investigate them separately. Therefore, the location where an attack may occur is considered as a dimension to classify the current works.

\section{B. Attack Impact on Transmitted Data}

In the security of cyber-physical systems, there are three properties that a system should possess for it to work properly, i.e., confidentiality, integrity and availability [10]. In the literature, different types of cyber attacks have been classified based on the properties that they threaten [10], [19]. For instance, deception attacks compromise the integrity, while denial of service attacks compromise the availability of the system [13]. Considering Figure 1, no matter what the type of the attack is, a sensor attack changes an event $\sigma \in \Sigma_{o v}$ in one of the following ways: deletion, insertion or replacement, where replacement can be viewed as composition of deletion and insertion. For instance, consider the example from [17] where $\Sigma=\Sigma_{o}=\{a, b, c\}, \Sigma_{o v}=\{a, c\}$ and the word $w=$ $a b b a$ executed in the plant. Then, considering all possibilities of deletion, insertion, and replacement under a sensor attack, the corrupted word belongs to $\{a, c\}^{*} b\{a, c\}^{*} b\{a, c\}^{*}$. An actuator attacker may affect a controllable vulnerable event $\sigma \in \Sigma_{c v}$ in one of the following ways: enablement or disablement. An actuator enablement attack can overwrite the supervisor's disablement action (for a vulnerable event) with an enablement action, and an actuator disablement attack can overwrite the enablement action with a disablement action. By replacement, we mean that the attacker may have both enablement and disablement impacts on a controllable vulnerable event. Since we focus on attack prevention, by definition, research works that deal with replacement attacks also deal with enablement attacks as well as disablement attacks.

For this reason, we assign one of the dimensions of our framework to attack impact on the information sent through communication channels, which are essentially attack mechanisms on these channels.

\section{Security Mechanism}

The last dimension differentiating between the existing research is the defense mechanism that they provide. Generally, an attack is successful if it causes damage to the system or brings it to an undesirable mode. With the purpose of preventing failure of a supervisory control system under attack, two main approaches have been presented in the literature:

1) detection and prevention,

2) synthesis of a resilient supervisor.

The first approach places an intrusion detection module (IDM) in the system to detect an attack and prevent it before it causes damage to the system by leading it to an unsafe state [18], [20]. The set of unsafe or critical states is assumed to be given, and they are mainly related to physical damage such as overflow or collision. As Figure 2 shows, the inserted module is connected to the supervisor and it observes the same events as the supervisor does. The module and the supervisor together will then form an integrated supervisor which determines control commands. To obtain such an IDM, first the behavior of the closed-loop control system under attack is modeled. The unsafe states of the closedloop control system are those indicating an unsafe state of the plant. The module is implemented such that it detects an attack leading to an unsafe state of the attacked closed-loop control system. In this case, it will force the supervisor to take a proper action either by disabling all controllable events 
as proposed in [20] or only those controllable events that can eventually cause the failure of the system as discussed in [17].

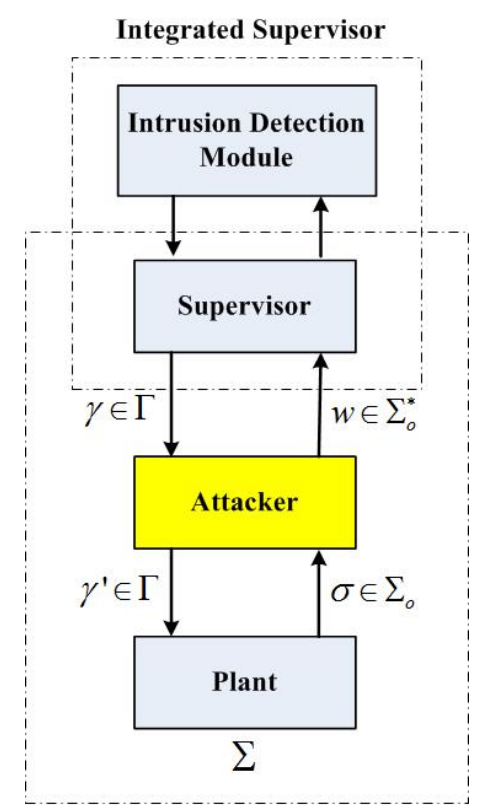

Closed-loop Control System

Fig. 2. Intrusion detection module approach

In the second approach, a supervisor is synthesized which is resilient against attacks. Based on the synthesis method, this approach can be divided into the following main subcategories:

- direct synthesis,

- reducing the problem to conventional synthesis.

In the first method, a new synthesis algorithm is provided to obtain a resilient supervisor. In [21], the author studies the problem of synthesis of resilient supervisors against sensor attacks. To solve the problem, first, a supremal successful attacker (an attacker that causes damage on the system) is synthesized, and based on that, a supervisor is synthesized which prevents system failure under attacks.

In the second method, conventional definitions of observability and controllability are first modified with respect to the effects of attacks on the system. Under the new conditions, the problem of supervisory control synthesis under attacks is reduced to the conventional synthesis problem [22].

Both general security approaches and each synthesis method will be considered in the classification as follows:

1) IDM

2) resilient supervisor: $\left\{\begin{array}{l}\text { new synthesis } \\ \text { conventional synthesis }\end{array}\right.$

\section{CLASSIFICATION}

To compare the existing approaches investigating attack prevention, we first classify them based on the proposed framework. The classification is presented in Figure 3.
There are other important aspects that the classification can also focus on. However, due to the limited number of research works that are currently available, not all possibilities of these aspects have been studied in the literature. We have thus made the decision not to include them in the classification. Nevertheless, we shall discuss these important aspects in Section VI.

\section{COMPARISON OF SECURITY APPROACHES}

In this section, we give an overview on advantages and disadvantages that each security approach brings. First, we focus on the two main approaches: IDM and resilient supervisory control synthesis. Then, we compare the two different directions that have been proposed for resilient supervisory control synthesis against attacks.

\section{A. IDM vs Synthesis}

The main difference between the existing IDM and resilient supervisor synthesis approach is that the first one critically relies on real-time attack detection and makes no assumption on the attacker, while the second approach constructs a supervisor so that the closed-loop system becomes not attackable, by using a prior knowledge of the attack models. Each mechanism has its own advantages and limitations depending on the application and the type of attack as discussed in the following.

1) Attack model: as we have explained before, in the IDM approach, no assumption is imposed on the attacker. The resilient synthesis approach imposes some assumptions on the attacker; in particular, it requires the attacker to know the model of the system. The assumption that the attacker knows the system model may seem a bit too strong. However, the assumption of a strong attacker is not necessarily bad as we focus on security and damage prevention. Indeed, in principle, if a resilient supervisor has been designed for an attacker that knows the system's model, then it will also be secure against attackers not knowing system's model. However, the existing resilient synthesis approach [21], [24], [25] also assumes the attacker tries to remain covert until causing guaranteed damage to the system. This additional assumption, which is not essential, limits the capability of the attacker (as no risk can be taken) and makes it harder to successfully attack the system. This limitation can be easily removed assuming a risky attacker, which we will discuss in Section VI.

2) Detectability: there are different types of attack strategies. An attack could be detectable if it changes the behavior of the system in such a way that it is distinguishable from the normal behavior. However, there exist more complex types of attacks which take the system model into consideration and use that knowledge to stay covert to the supervisor until the damage is caused by attacks. The implementation of an IDM is limited to systems satisfying the detectability condition, i.e., systems in which an attack can be detected before it causes any damage to the system by leading it to an unsafe state [18], [20]. The detectability condition has been further modified in [17] so that an attack is undetectable 


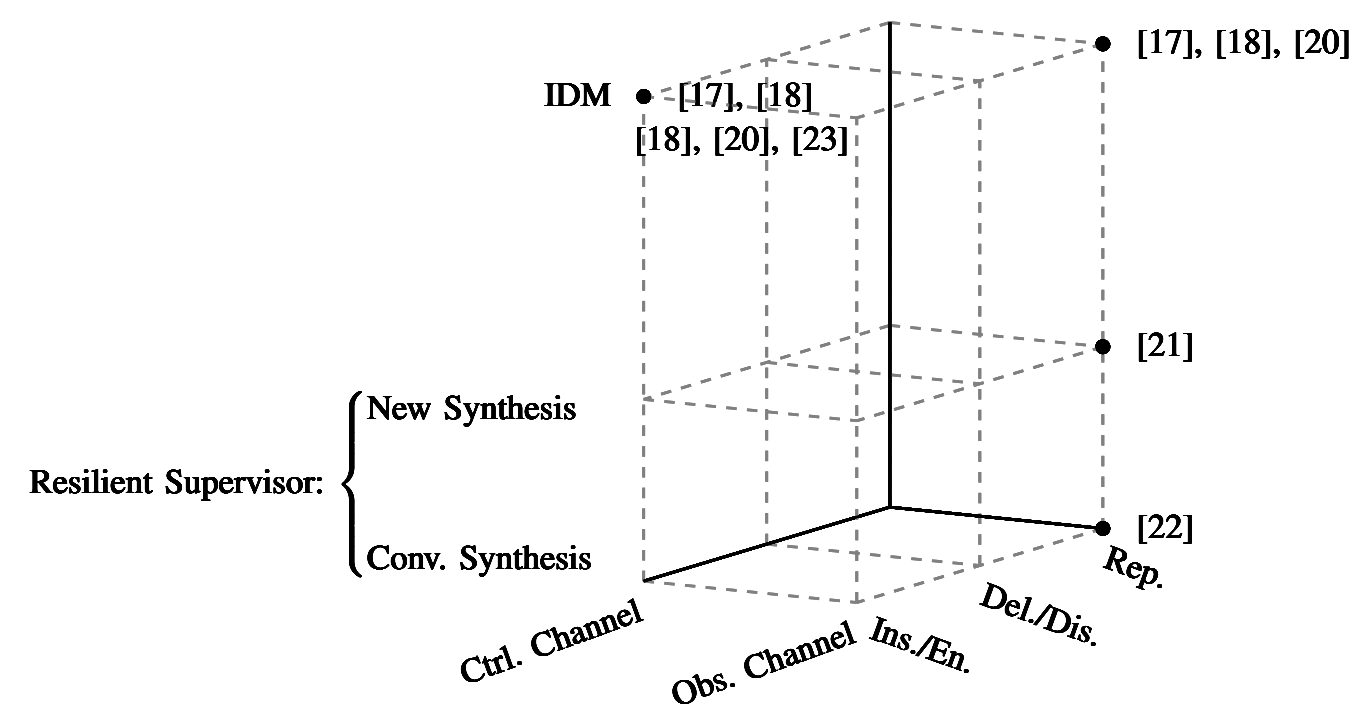

Fig. 3. Classification of approaches on attack-prevention in supervisory control systems in the proposed framework.

until reaching an unsafe boundary but may be detected after it reaches the boundary. While undetectable attacks cannot be handled by IDM, most of the resilient supervisor synthesis approaches focus on attacks which cannot be detected until causing damage [21], [22], [26].

3) Closed-loop system behavior: As long as no attack has occurred, the IDM approach preserves the original closedloop system behavior, while the resilient supervisor synthesis approach has to restrict the behavior of the closed-loop system from the beginning (in order to make it harder to attack), which limits the permissiveness of the supervisor. In the resilient supervisor synthesis approach, the supervisor can even be designed such that enablement attack may become impossible (in the actuator attack scenario) and thus normal execution may not have to be terminated ${ }^{3}$ (depending on the permissiveness of the synthesized supervisor). The IDM approach has to terminate normal execution after detecting an attack. Compared with the resilient supervisor synthesis approach, instead of replacing all control logic implemented in the system, the IDM approach only needs to design and integrate an additional security module.

4) Defense philosophy: the defense philosophy in the IDM approach is quite straightforward. It relies solely on attack detection for preventing damage caused by attacks. This attack detection mechanism also exists in the synthesis approach, but a much richer defense philosophy can co-exist in the synthesis approach, due to the various assumptions imposed. For example, in the resilient synthesis approach, the attacker may be assumed to try to remain covert in its initial attacks before it can cause damage to the system in its final attack [21], [24]. This assumption on the attacker can be used to design supervisors that discourage the attacker from attacking the system. In particular, different assumptions on

\footnotetext{
${ }^{3}$ For example, an extremely conservative approach is to treat each plant state with a vulnerable event defined as a bad state in the synthesis of the supervisor. Then, there is even no possibility of attack!
}

the attacker's model can lead to different defense strategies. These features do not exist in current implementations of the IDM approach.

\section{B. New Synthesis vs. Conventional Synthesis}

Here, we shall discuss the benefits and limitations of each method for resilient supervisory control synthesis, as far as the current research works are concerned. First of all, reducing the problem of supervisory control synthesis under attacks to conventional synthesis brings the advantage of being able to use the available techniques and tools. However, in [22], new conditions of controllability and observability are essentially proposed only for verification and thus they are quite restrictive. This is a limitation of [22], but it does not seem to be a fundamental limitation of the reduction-based approach. As presented in [21], the direct synthesis approach involves a two-step synthesis since a supremal successful attacker needs to be synthesized first, which is then used to synthesize a resilient supervisor. In order to synthesize a supremal successful attacker, a normality assumption is imposed [21], [24]. This assumption is relaxed in [26], and they construct an attacker allowing a larger class of attack strategies. Although the attacker is supposed to be at the basis to finding a resilient supervisor, this has not yet been provided in [24], [26]. Moreover, the resilient supervisor synthesis algorithm provided in [21] is sound but not complete. That is, it is possible that there exists a resilient supervisor but the algorithm in [21] fails to find one.

\section{RELATED WORKS}

Besides papers working on security and resilience against attacks, there are works on robust supervisory control against similar effects as attacks. These papers are briefly discussed here as they may contain ideas that can help us solve existing problems in the attack-prevention domain or inspire 
new research works. As we do not intend to conduct a comprehensive review of these related works, the reader is referred to the cited papers for more details.

\section{A. Supervisory Control under Communication Problems}

The effects of attacks could be the same as the effects of other communication problems on supervisory control systems. For instance, communication losses can be modeled and dealt with in the same way as deletion of vulnerable events sent through communication channels [27][30]. Moreover, the effects of communication delays can be modeled in the same way as deletion and insertion of vulnerable events. For instance, let us consider that an event, say $\sigma \in \Sigma_{o}$, is observed after a delay of one step (of the event occurrence), then in the delayed observed plant, $\sigma$ is deleted at every state where it is enabled and inserted after the next transition. There are several papers investigating networked supervisory control of discrete-event systems with communication problems such as delays, losses and nonFIFO observations [31], [32]. To deal with network communication delays and losses, in [31], a mapping is introduced to model the plant under observation delay and losses. To synthesize a networked supervisor, they provide new controllability and observability conditions under delay and losses called network controllability and network observability. For a plant and requirements satisfying network controllability and network observability conditions, a networked supervisor is obtained by applying the conventional synthesis.

Moreover, in [32], a non-FIFO observation channel is considered where events may be observed not necessarily in the same order as they have been executed in the plant. For instance, a word $w=\sigma_{1} \sigma_{2}$ where $\sigma_{1}, \sigma_{2} \in \Sigma_{o}$ may be observed as $\sigma_{1} \sigma_{2}$ or $\sigma_{2} \sigma_{1}$. Non-FIFO observation has the same effect as a replacement attack in the sensor channel. In [32], a networked supervisor which is robust to communication delays and non-FIFO observations is synthesized through a new synthesis algorithm.

\section{B. Fault-tolerant Supervisory Control of Discrete Event Sys- tems}

The impact of faults on supervisory control of a DES might be more general than deletion and insertion of vulnerable events in the attack domain. A fault may map the nominal behavior of the supervised system to a faulty behavior. However, fault-tolerant supervisory control approaches [33] use quite similar mechanisms to deal with the effects of faults which can be divided as:

- active fault tolerant supervisory control such as in [34], [35],

- passive fault tolerant supervisory control such as in [36], [37].

The first method is based on real-time fault detection and prevention. The strategy is the same as in the IDM approach. If a system satisfies diagnosability conditions, then a diagnoser is implemented. The diagnoser detects the occurrence of a fault before the system executes some illegal sequences. The nominal supervisor will then be switched to a new supervisor to satisfy some post-fault specifications [35]. A fault diagnoser cannot be implemented for applications which do not satisfy diagnosability conditions.

The second approach provides a fault-tolerant supervisory control synthesis technique as discussed in [36]. In this method, first, the nominal behavior of the plant and the faulty behavior are modeled as a fault-accommodating behavior. Using this model, an acceptable behavior is defined based on the nominal desired behavior and the defective acceptable behavior. As a result, the fault-tolerant supervisory control problem is transformed to a standard supervisory control problem under partial observations for which existing algorithms and tools can be used as discussed in [36]. This approach is limited to faults occurring only once and with a certain degradation behavior.

\section{Attacker Synthesis}

Furthermore, there are a few papers that only investigate attacker synthesis, as we have discussed before. For instance, in [26], [38], a successful insertion-deletion undetectable sensor attack is constructed and in [24], actuator attacks have been modeled under the assumption of a normality condition on the attacker and supervisor, in which case the supremal successful actuator attacker exists. In particular, under normality assumption on the supervisor, the attacker can exercise enablement attack for at most once. Moreover, under normality assumption on the attacker, disablement attack does not help enablement attack in achieving the attack goal, with the aim of remaining covert.

\section{TOPICS TO INVESTIGATE}

All the discussed papers follow the main objective of presenting a security approach that can handle most kinds of attacks, at least in principle. To achieve this goal, the current techniques still need to be improved in the following aspects.

\section{A. Actuator Disablement Attack and Replacement Attack}

As Figure 3 reveals, papers investigating synthesis of resilient supervisors have focused only on sensor attacks. [24] provides an algorithm for the synthesis of a supremal successful actuator attacker under a normality assumption; however, no resilient supervisory control synthesis algorithm has been provided there. The problem has only been partially addressed in [39], [40], by solving an alternative problem of supervisor obfuscation. IDM approaches only considered enablement actuator attacks for the reason that disablement does not lead the system to an unsafe state, and it may only cause blocking issues (which are not important compared to security). On the other hand, it has been remarked in [24] that disablement attacks, in addition to enablement attacks, could be critical for a successful actuator attacker under the assumption that the attacker would like to remain covert. With a normality assumption imposed on the supervisor and attacker [24], it has been shown that a replacement attacker is no more powerful than an enablement attacker. No synthesis algorithm has been provided for actuator replacement attacks 
for the general case without the normality assumption, and no resilient supervisor synthesis algorithm has been provided for the general case as well.

\section{B. Attacks with Delay/Disordering Impact}

All the available works assume that the attacker modifies the transmitted data instantaneously, and also it does not change the relative ordering of data (events). However, an attacker may add delay in communication. It also may change the ordering of events sent through the same or different communication channels (either observation or control channels). Therefore, delaying and disordering of transmitted data still need to be investigated as other attack impacts besides insertion and deletion (or enablement and disablement).

\section{Non-risky versus risky attackers}

A non-risky attacker carries out an attack only if 1) it remains covert after the attack, or 2) the attack will cause guaranteed damage to the system [21], [24]. On the other hand, being covert is typically not a goal of a risky attacker [26]. While most of the papers have focused on nonrisky attackers, there could be scenarios in which an attacker is risky. In particular, a risky attacker implements a worstcase attack scenario and resilient supervisors against risky attackers tend to have less permissiveness. A risky sensor attacker has been synthesized in [26]. However, there still does not exist any supervisor handling this type of attack.

\section{Active Attacker with Partial Observation of $\Sigma_{o}$}

An intelligent attacker (undetectable) can perform a successful attack based on its knowledge of the system model and its observation on the execution of the closed-loop system. However, an attacker may not always have full observation of $\Sigma_{o}$. In [24], a successful actuator attacker is synthesized that can only observe a subset of $\Sigma_{o}$, under the assumption that the attacker can observe the control command issued by the supervisor each time when the supervisor observes an event $\sigma \in \Sigma_{o}$. Based on the newly issued control command, the attacker can infer that some observable event of the supervisor has already been fired, even if that event is unobservable to the attacker. Moreover, the attacker and the supervisor satisfy a normality assumption, that is, all vulnerable events are observable to the attacker and all controllable events are observable to the supervisor. These conditions can be relaxed. In addition, the supervisor may not issue a control command each time when it observes an execution of $\sigma \in \Sigma_{o}$, but instead it only sends the control command message when it is necessary, which makes the closed-loop system harder to attack. No resilient supervisor synthesis algorithm is available under this condition.

\section{E. Passive Attacks and Opacity Enforcement}

As we have discussed, we have mainly focused on active attacks, that is, the attacker's goal is to inflict damage on the system. There is a more benign class of attacks, where the attacker's goal is to compromise the confidentiality of the system and learn secrets. An important notion for this purpose is opacity.
Opacity is a cyber-security property that recently has gained a lot of attention in supervisory control of DES [41][50]. A system is opaque if an outside observer, who knows the model of the system but has only partial observation on the system evolution, cannot infer a "secret" about the system behavior [47], [51]. In other words, the secret behavior is observationally equivalent to the non-secret behavior for the intruder. Opacity can be enforced either by an external enforcer or by synthesizing an opacity-enforcing supervisor as discussed in [45], [52]-[54] and in [46], [51], respectively.

Consider an attacker that uses the knowledge of the system model to perform a successful covert attack, based on its partial observation on the execution of the system. If we make the unsafe behavior (strings from which the system can reach an unsafe state) opaque to the attacker, then the attacker may not be able to see an opportunity to make a successful attack. Securing a networked supervisory control system using opacity enforcement techniques is an ongoing research topic [49].

\section{F. Supervisor Obfuscation}

As an alternative method for resilient supervisor synthesis, supervisor obfuscation can be used. For instance, in [39], an algorithm is proposed to obfuscate an insecure supervisor to make it resilient against actuator attacks, while preserving the behavior of the original closed-loop system. An application of this approach is to first synthesize a supervisor that takes care of all the safety specifications, except for the resilience property; the insecure supervisor can then be obfuscated to become resilient. This makes supervisor obfuscation another two-step synthesis method. However, a limitation of the algorithm in [39] for solving the supervisor obfuscation problem is that it is sound but incomplete. Moreover, the efficiency of the proposed supervisor obfuscation algorithm in [39] needs to be improved.

\section{G. Other Topics}

There are many other important topics that we are not able to cover here, including (probabilistic) attack on probabilistic systems, game based solving approach, model checking based solving approach, ideas using attack trees and graphs [55]-[58] and so on. In addition, it is possible to investigate cooperative attack scenarios or decentralized attack architectures in similar spirit to [59], [60]. Last but not the least, it is important to consider the potential applications for securing practical industrial control systems. Towards this goal, a set of benchmark examples (see, for example, [61]) that mimic industrial control systems can be constructed for the researchers to test and compare the techniques and tools.

\section{CONCLUSIONS}

In this paper, we have classified the current research works in the attack prevention of supervisory control systems using a framework consisting of the following dimensions: 1) the communication channel which is exposed to an attacker, 2) the attack impact on the transmitted data, and 3) the defense mechanism. We have also surveyed other relevant 
works investigating similar problems such as robust supervisory control approaches against faults, communication delays, losses and non-FIFO observations. Finally, we have pinpointed some under-explored topics for further investigations. Based on the discussions, we can conclude that this research topic is currently attracting a lot of attention from researchers in supervisory control community, and many technical problems still need to be solved.

\section{REFERENCES}

[1] J. Shi, J. Wan, H. Yan, and H. Suo, "A survey of cyber-physical systems," in Wireless Communications and Signal Processing (WCSP), 2011 International Conference on. IEEE, 2011, pp. 1-6.

[2] E. Ciancamerla, B. Fresilli, M. Minichino, T. Patriarca, and S. Iassinovski, "An electrical grid and its scada under cyber attacks: Modelling versus a hybrid test bed," in 2014 International Carnahan Conference on Security Technology (ICCST), Oct 2014, pp. 1-6.

[3] S. Sridhar, A. Hahn, M. Govindarasu et al., "Cyber-physical system security for the electric power grid." Proceedings of the IEEE, vol. 100, no. 1, pp. 210-224, 2012.

[4] F. Pasqualetti, F. Dörfler, and F. Bullo, "Cyber-physical attacks in power networks: Models, fundamental limitations and monitor design," in Decision and Control and European Control Conference (CDCECC), 2011 50th IEEE Conference on. IEEE, 2011, pp. 2195-2201.

[5] Q. Zhu and T. Başar, "Robust and resilient control design for cyberphysical systems with an application to power systems," in Decision and Control and European Control Conference (CDC-ECC), 2011 50th IEEE Conference on. IEEE, 2011, pp. 4066-4071.

[6] A. Teixeira, H. Sandberg, and K. H. Johansson, "Networked control systems under cyber attacks with applications to power networks," in Proceedings of the 2010 American Control Conference, 2010, pp. 3690-3696.

[7] N. Nicolaou, D. G. Eliades, C. Panayiotou, and M. M. Polycarpou, "Reducing vulnerability to cyber-physical attacks in water distribution networks," in 2018 International Workshop on Cyber-physical Systems for Smart Water Networks (CySWater). IEEE, 2018, pp. 16-19.

[8] S. Amin, X. Litrico, S. S. Sastry, and A. M. Bayen, "Stealthy deception attacks on water scada systems," in Proceedings of the 13th ACM international conference on Hybrid systems: computation and control. ACM, 2010, pp. 161-170.

[9] D. G. Eliades and M. M. Polycarpou, "A fault diagnosis and security framework for water systems," IEEE Transactions on Control Systems Technology, vol. 18, no. 6, pp. 1254-1265, 2010.

[10] A. A. Cardenas, S. Amin, and S. Sastry, "Secure control: Towards survivable cyber-physical systems," in Distributed Computing Systems Workshops, 2008. ICDCS'08. 28th International Conference on. IEEE, 2008, pp. 495-500.

[11] A. Cardenas, S. Amin, B. Sinopoli, A. Giani, A. Perrig, S. Sastry et al., "Challenges for securing cyber physical systems," in Workshop on future directions in cyber-physical systems security, vol. 5, 2009.

[12] H. Fawzi, P. Tabuada, and S. Diggavi, "Secure estimation and control for cyber-physical systems under adversarial attacks," IEEE Transactions on Automatic Control, vol. 59, no. 6, pp. 1454-1467, 2014.

[13] F. Pasqualetti, F. Dörfler, and F. Bullo, "Attack detection and identification in cyber-physical systems," IEEE Transactions on Automatic Control, vol. 58, no. 11, pp. 2715-2729, 2013.

[14] C. G. Cassandras and S. Lafortune, Introduction to discrete event systems. Springer Science \& Business Media, 2009.

[15] P. J. Ramadge and W. M. Wonham, "Supervisory control of a class of discrete event processes," SIAM Journal on Control and Optimization, vol. 25, no. 1, pp. 206-230, 1987.

[16] M. Uma and G. Padmavathi, "A survey on various cyber attacks and their classification." IJ Network Security, vol. 15, no. 5, pp. 390-396, 2013.

[17] P. M. Lima, L. K. Carvalho, and M. V. Moreira, "Detectable and undetectable network attack security of cyber-physical systems," IFACPapersOnLine, vol. 51, no. 7, pp. 179-185, 2018.

[18] L. K. Carvalho, Y.-C. Wu, R. Kwong, and S. Lafortune, "Detection and mitigation of classes of attacks in supervisory control systems," Automatica, vol. 97, pp. 121-133, 2018.
[19] A. Teixeira, D. Pérez, H. Sandberg, and K. H. Johansson, "Attack models and scenarios for networked control systems," in Proceedings of the 1st International Conference on High Confidence Networked Systems. New York, NY, USA: ACM, 2012, pp. 55-64.

[20] P. M. Lima, M. V. Alves, L. K. Carvalho, and M. V. Moreira, "Security against network attacks in supervisory control systems," IFAC-PapersOnLine, vol. 50, no. 1, pp. 12333-12 338, 2017.

[21] R. Su, "Supervisor synthesis to thwart cyber attack with bounded sensor reading alterations," Automatica, vol. 94, pp. 35-44, 2018.

[22] M. Wakaiki, P. Tabuada, and J. P. Hespanha, "Supervisory control of discrete-event systems under attacks," Dynamic Games and Applications, pp. 1-19, 2017.

[23] D. Thorsley and D. Teneketzis, "Intrusion detection in controlled discrete event systems," in Decision and Control, 2006 45th IEEE Conference on. IEEE, 2006, pp. 6047-6054.

[24] L. Lin, S. Thuijsman, Y. Zhu, S. Ware, R. Su, and M. Reniers, "Synthesis of successful actuator attackers on supervisors," arXiv preprint arXiv:1807.06720, 2018.

[25] — - "Synthesis of supremal successful normal actuator attackers on normal supervisors," American Control Conference, 2018.

[26] R. M. Góes, E. Kang, R. Kwong, and S. Lafortune, "Stealthy deception attacks for cyber-physical systems," in Decision and Control (CDC), 2017 IEEE 56th Annual Conference on. IEEE, 2017, pp. 4224-4230.

[27] M. V. S. Alves, J. C. Basilio, A. E. C. da Cunha, L. K. Carvalho, and M. V. Moreira, "Robust supervisory control against intermittent loss of observations," IFAC Proceedings Volumes, vol. 47, no. 2, pp. 294-299, 2014.

[28] K. Rohloff, "Bounded sensor failure tolerant supervisory control." in WODES, 2012, pp. 272-277.

[29] T. Ushio and S. Takai, "Nonblocking supervisory control of discrete event systems modeled by mealy automata with nondeterministic output functions," IEEE Transactions on Automatic Control, vol. 61, no. 3, pp. 799-804, 2016.

[30] X. Yin, "Supervisor synthesis for mealy automata with output functions: A model transformation approach," IEEE Transactions on Automatic Control, vol. 62, no. 5, pp. 2576-2581, 2017.

[31] F. Lin, "Control of networked discrete event systems: dealing with communication delays and losses," SIAM Journal on Control and Optimization, vol. 52, no. 2, pp. 1276-1298, 2014.

[32] A. Rashidinejad, M. Reniers, and L. Feng, "Supervisory control of timed discrete-event systems subject to communication delays and non-fifo observations," IFAC-PapersOnLine, vol. 51, no. 7, pp. $456-$ 463, 2018, 14th IFAC Workshop on Discrete Event Systems WODES 2018.

[33] T. Moor, "A discussion of fault-tolerant supervisory control in terms of formal languages," Annual Reviews in Control, vol. 41, pp. 159-169, 2016.

[34] A. Paoli and S. Lafortune, "Safe diagnosability for fault-tolerant supervision of discrete-event systems," Automatica, vol. 41, no. 8, pp. 1335-1347, 2005.

[35] A. Paoli, M. Sartini, and S. Lafortune, "Active fault tolerant control of discrete event systems using online diagnostics," Automatica, vol. 47, no. 4, pp. 639-649, 2011.

[36] T. Wittmann, J. Richter, and T. Moor, "Fault-tolerant control of discrete event systems based on fault-accommodating models," IFAC Proceedings Volumes, vol. 45, no. 20, pp. 854-859, 2012.

[37] S. Shu and F. Lin, "Fault-tolerant control for safety of discrete-event systems," IEEE Transactions on Automation Science and Engineering, vol. 11, no. 1, pp. 78-89, 2014.

[38] Q. Zhang, Z. Li, C. Seatzu, and A. Giua, "Stealthy attacks for partially-observed discrete event systems," in 2018 IEEE 23rd International Conference on Emerging Technologies and Factory Automation (ETFA), vol. 1. IEEE, 2018, pp. 1161-1164.

[39] Y. Zhu, L. Lin, and R. Su, "Supervisor obfuscation against actuator enablement attack," in arXiv:1811.02932v1, 2018.

[40] — - "Supervisor obfuscation against actuator enablement attack," in European Control Conference, 2018.

[41] B. Bérard, J. Mullins, and M. Sassolas, "Quantifying opacity," Mathematical Structures in Computer Science, vol. 25, no. 2, pp. 361-403, 2015.

[42] A. Saboori and C. N. Hadjicostis, "Notions of security and opacity in discrete event systems," in Decision and Control, 2007 46th IEEE Conference on. IEEE, 2007, pp. 5056-5061.

[43] F. Lin, "Opacity of discrete event systems and its applications," Automatica, vol. 47, no. 3, pp. 496-503, 2011. 
[44] A. Saboori and C. N. Hadjicostis, "Opacity-enforcing supervisory strategies via state estimator constructions," IEEE Transactions on Automatic Control, vol. 57, no. 5, pp. 1155-1165, 2012.

[45] Y.-C. Wu and S. Lafortune, "Synthesis of insertion functions for enforcement of opacity security properties," Automatica, vol. 50, no. 5, pp. 1336-1348, 2014.

[46] X. Yin and S. Lafortune, "A uniform approach for synthesizing property-enforcing supervisors for partially-observed discrete-event systems," IEEE Transactions on Automatic Control, vol. 61, no. 8, pp. 2140-2154, 2016.

[47] R. Jacob, J.-J. Lesage, and J.-M. Faure, "Overview of discrete event systems opacity: Models, validation, and quantification," Annual reviews in control, vol. 41, pp. 135-146, 2016.

[48] S. Lafortune, F. Lin, and C. N. Hadjicostis, "On the history of diagnosability and opacity in discrete event systems," Annual Reviews in Control, vol. 45, pp. 257-266, 2018.

[49] X. Yin and S. Li, "Verification of opacity in networked supervisory control systems with insecure control channels," in 57th IEEE Conference on Decision and Control. IEEE, Accepted, 2018.

[50] J. Dubreil, P. Darondeau, and H. Marchand, "Supervisory control for opacity," IEEE Transactions on Automatic Control, vol. 55, no. 5, pp. 1089-1100, 2010.

[51] Y. Tong, Z. Li, C. Seatzu, and A. Giua, "Current-state opacity enforcement in discrete event systems under incomparable observations," Discrete Event Dynamic Systems, vol. 28, no. 2, pp. 161-182, 2018.

[52] F. Cassez, J. Dubreil, and H. Marchand, "Synthesis of opaque systems with static and dynamic masks," Formal Methods in System Design, vol. 40, no. 1, pp. 88-115, 2012.

[53] Y. Ji, Y.-C. Wu, and S. Lafortune, "Enforcement of opacity by public and private insertion functions," Automatica, vol. 93, pp. 369-378, 2018.

[54] X. Yin and S. Li, "Synthesis of dynamic masks for infinite-step opacity," IFAC-PapersOnLine, vol. 51, no. 7, pp. 343-348, 2018.

[55] M. Svoreňová and M. Kwiatkowska, "Quantitative verification and strategy synthesis for stochastic games," European Journal of Control, vol. 30, pp. 15-30, 2016.

[56] S. Jha, O. Sheyner, and J. Wing, "Two formal analyses of attack graphs," in Computer Security Foundations Workshop, 2002. Proceedings. 15th IEEE. IEEE, 2002, pp. 49-63.

[57] S. A. Camtepe and B. Yener, "Modeling and detection of complex attacks," in Security and Privacy in Communications Networks and the Workshops, 2007. SecureComm 2007. Third International Conference on. IEEE, 2007, pp. 234-243.

[58] B. Kordy, L. Piètre-Cambacédès, and P. Schweitzer, "Dag-based attack and defense modeling: Dont miss the forest for the attack trees," Computer science review, vol. 13, pp. 1-38, 2014.

[59] Y. Tong, K. Cai, and A. Giua, "Decentralized opacity enforcement in discrete event systems using supervisory control," in 2018 57th Annual Conference of the Society of Instrument and Control Engineers of Japan (SICE), 2018, pp. 1053-1058.

[60] B. Wu, J. Dai, and H. Lin, "Synthesis of insertion functions to enforce decentralized and joint opacity properties of discrete-event systems," in 2018 Annual American Control Conference (ACC). IEEE, 2018, pp. 3026-3031.

[61] E. Kang, S. Adepu, D. Jackson, and A. P. Mathur, "Model-based security analysis of a water treatment system," in 2016 IEEE/ACM 2nd International Workshop on Software Engineering for Smart CyberPhysical Systems (SEsCPS), 2016, pp. 22-28. 